\title{
Some Spanners in the Works of Grounding Mechanisms Removed
}

\section{Robin Stenwall ${ }^{1}$ (D)}

Received: 1 July 2021 / Accepted: 1 October 2021 / Published online: 18 October 2021

(c) The Author(s) 2021

\begin{abstract}
In this paper I address two concerns with Kelly Trogdon's grounding mechanism view, i.e. the idea that metaphysical explanation can be modeled on causal-mechanical explanation. The first concern threatens to undermine the unity that groundingmechanical explanations imposes on metaphysical explanation; and the second concern requires the grounding mechanic to put forth a formal condition on grounding-mechanical models. After having discussed both of these, I provide a solution to the first and argue that the second concern is unwarranted.
\end{abstract}

Keywords Metaphysical explanation · Metaphysical grounding $\cdot$ Mechanisms · Causation

\section{Grounding-Mechanical Explanations}

Kelly Trogdon (2018) has put forth a theory on how to model metaphysical explanation on causal-mechanical explanation that he calls the grounding mechanism view. The idea is that "some metaphysical explanations are representations of grounding relations as being instances of grounding mechanisms" (Trogdon 2018: 1296). The explanandum of a grounding-mechanical explanation is the obtaining of a grounded fact, and just as we can explain a phenomenon by providing a model of the inner workings of the mechanism that produces the phenomenon (Machamer et al. 2000), we can explain why a grounded fact obtains by providing a model of "how the

Some spanners in the works of grounding mechanisms removed.

Robin Stenwall

robin.stenwall@fil.lu.se

1 Department of Philosophy, Lund University, Lund, Sweden 
connection runs between grounding facts and facts they ground" (Trogdon 2018: 1298, emphasis added).

Like their causal counterparts, grounding mechanisms are decompositional in that they can be broken down into constituents of facts. ${ }^{1}$ Assuming that facts are complex entities, Trogdon takes the constituents of facts to be objects and properties. The constituents of grounding facts are said to stand in metaphysical determination relations to the constituents of the facts they ground. Thus, suppose the following condition holds true of some relation $\mathrm{R}$ :

Grounding Condition: if the $x$ s stand in $\mathrm{R}$ to $y$ then there is some fact A, some fact $\mathrm{B}$, and some plurality of facts $\Delta$ such that the $x$ s are constituents of $\mathrm{A}, y$ is a constituent of $\mathrm{B}, \mathrm{A}$ is among $\Delta$, and $\Delta$ grounds $\mathrm{B}$ (Trogdon 2018: 1291). ${ }^{2}$

Trogdon stipulates that $\mathrm{R}$ is a metaphysical determination relation if and only if the above condition is an essential truth about $\mathrm{R}$. Relations like set formation, the determinate-determinable relation, functional realization, constitution, truthmaking and mereological realization are all said to be examples of relations among the constituents of facts that are essentially connected to the Grounding Condition (Trogdon 2018: 1291-2 and Trogdon 2020: 396-407). Thus, for example, it is essential to set formation that, if some object exist, then the fact that it exists grounds the fact that the singleton of the object exists. Similarly, it is essential to truthmaking that if Socrates stands in the truthmaking relation to (Socrates exists) then [Socrates exists] grounds the alethic fact that (Socrates exists $\rangle$ is true (Trogdon 2020: 404). ${ }^{3}$ In both cases the grounding is said to be full in that there are no facts to be added to the grounding facts for them to ground what they ground. There are, however, other metaphysical determination relations where the corresponding grounding facts are mere partial grounds in that they need to be supplemented with additional facts to fully ground what they ground. Trogdon mentions constitution, functional realization and mereological realization as examples of such determination relations. Thus, it is essential to functional realization that if $\mathrm{Q}$ is functionally realized by $\mathrm{P}$ when $\mathrm{x}$ is $\mathrm{P}$ and $\mathrm{Q}$, then $[\mathrm{x}$ is $\mathrm{P}]$ is among some plurality of facts $\Gamma$, such that $\Gamma$, rather than [x is P] alone, grounds [x is Q] (Trogdon 2018: 1292).

Trogdon characterizes grounding mechanisms in terms of such relations. A grounding mechanism, he says, is either a metaphysical determination relation or a chain of such relations. Thus, assuming that some plurality of facts $\Delta$ grounds A, this is an instance of a grounding mechanism just in case there is some fact among $\Delta$ with a constituent(s) that stands in a metaphysical determination relation or a chain of such relations to some constituent of A (Trogdon 2018: 1293). Such mechanisms can be more or less complex depending on how many chains link the constituents

\footnotetext{
1 Causal mechanisms are thought to be decompositional in the sense that the overall behaviour of a mechanism can be broken down into complex arrangements among the activities of the parts of the mechanism (Craver 2007).

2 The plurality is here allowed to have just one member.

${ }^{3}$ I follow the convention of using square brackets to form names of facts and angle brackets to form names of propositions. Thus " $[\mathrm{P}]$ " denotes the fact that $\mathrm{P}$, whereas " $(\mathrm{P}\rangle$ " denotes the proposition that $\mathrm{P}$.
} 
of the grounding fact with the constituents of the grounded fact. In the simplest case, like when [Socrates exists] grounds [ $\{$ Socrates $\}$ exists], there is a single metaphysical determination relation of set formation linking Socrates to his singleton. In other, more complex cases, the grounding is mediated through chains of determination relations as, for example, when Socrates stands in the relation of set formation to $\{$ Socrates\}; and the singleton, in turn, stands in the truthmaking relation to ( $\{$ Socrates $\}$ exists $\rangle$ thereby accounting for how the connection runs between the fact that Socrates exists and the fact that the proposition stating that $\{$ Socrates exists is true (Trogdon 2020: 404).

The main virtue of the grounding mechanism view is that it allows us to evaluate the plausibility of various grounding-theoretic theses. A grounding-mechanical model, on this view, explains why a grounded fact obtains by revealing the metaphysical determination relations that hold between the constituents of the relevant facts (for a list of examples, see Trogdon 2018: 1297-99). Some grounding claims seem implausible simply because it is not clear how the grounding connection is meant to work. As an example, consider the following:

[Kelly loves Gomer] grounds [\{Socrates $\}$ exists].

The claim seems implausible because we don't know of any model that correctly depicts the determination relations at play between the constituents of the fact involving Kelly and his dog Gomer on the one hand and the fact involving the singleton on the other. Similar mechanistic objections can be raised against priority monism (Schaffer 2010), mathematical structuralism (Shapiro 1997) and physicalism (Levine 2001), simply because we lack plausible models that tell us about the metaphysical determination relations that are operative in each case (Trogdon 2018: 1303-06).

Another virtue of the view is that it allows the grounding theorist to provide further justification to the conclusion that grounding is in some important sense unified. The standard way to argue for unification is to appeal to formal properties- the idea being that various fine-grained determination relations share certain formal features (e.g. being strict partial orderings ${ }^{4}$ and non-monotonic) that unify them. ${ }^{5}$ By employing the grounding mechanism view, Trogdon thinks that the grounding theorist is able to further strengthen her case against the grounding skeptic by pointing to the fact that determination relations are unified by all being connected to grounding in the same way, namely by essentially satisfying the Grounding Condition (2018: 1292-93). ${ }^{6}$

\footnotetext{
4 That grounding is a strict partial ordering remains the orthodox view, although the claim has been subject to counterexamples by Fine (2010); Schaffer (2012); Krämer (2013) and Tahko (2013) (although, see Raven (2013); Litland (2013) and Rodriguez-Pereyra (2015) for why some of these counterexamples are unsuccessful). I will follow the orthodoxy as this induces a metaphysical structure that allows me to identify the fundamental facts with the minimal elements of the ordering.

5 Although, see Koslicki 2015 and Wilson 2016.

${ }^{6}$ As a matter of fact, if Trogdon is correct, then grounding is not only unified but plays an indispensable role in various metaphysical explanations (although, see Maurin 2019).
} 
I'm largely sympathetic to the recent trend among metaphysicians to model metaphysical explanation on pre-existing and well-established theories of explanation within the sciences ${ }^{7}$ - not least, because by emulating one has the chance to increase one's understanding of a phenomenon without having to invent a completely new theory of explanation. I'm also sympathetic to the idea of emulating on the causalmechanical model of explanation in particular as it forces the grounding theorist to reveal the more fine-grained connections that underlie the obtaining of facts. Having said that, I also think that more needs to be done with the view if it is to be a unified theory of a certain kind of metaphysical explanation. In Sects. 2. and 3., I discuss two concerns that Trogdon raises and that pertain to the kind of unity at hand. At the end of Sect. 3. I argue that the second concern is unwarranted, focusing on how to deal with the first, and more severe, concern in Sect. 4. Section 5. summarizes the discussion.

\section{Grounding unaccompanied by grounding mechanisms}

Some metaphysical explanations cannot straightforwardly be understood in terms of ground (e.g. reductive and essentialist explanation). ${ }^{8}$ Not surprisingly, the grounding mechanism view cannot handle such explanations, as there will be no corresponding instances of grounding mechanisms to represent. Trogdon acknowledges this, but insists on the weaker mechanical backing claim, namely that "any metaphysical explanation that is backed by grounding-any metaphysical explanation that proceeds by way of linking grounds to what they ground-is a grounding-mechanical explanation" (Trogdon 2018: 1303). The result is a unified theory of explanation, at least of metaphysical explanations that work by being backed by grounding.

But is this really true? Consider the following claim:

[ $a$ is green] and [ $b$ is blue] grounds [ $a$ is green and $b$ is blue],

and suppose that it is true. If all there is to a mechanism is a metaphysical determination relation or a chain of such relations, then there is no grounding mechanism present in this case-this, since there are no metaphysical determination relations that connect constituents of either conjunct fact to the constituents of the conjunctive fact. It is a matter of logic alone that if $[\mathrm{F} a]$ obtains and [G $b]$ obtains, then $\left[\mathrm{F} a^{\wedge} \mathrm{G} b\right]$ obtains. It is similarly a matter of logic that if $[\mathrm{Fa}]$ obtains, then so do both $\left[\mathrm{F} a^{\vee} \mathrm{G} b\right]$ and $[\exists \mathrm{x} F(\mathrm{x})]$. But of course, rules of inference like existential generalization, conjunction and disjunction introduction, if relations at all (as opposed to operations), are not metaphysical determination relations, as they would have to take propositions or sentences, rather than objects or properties as their relata (Trogdon

\footnotetext{
7 See e.g. Wilsch (2016) for an attempt to understand metaphysical explanation as a type of nomological explanation; Baron \& Norton (2021) and Kovacs (2020) for an attempt to model metaphysical explanation along unificationist lines; and Miller \& Norton (2017) and Thompson (2019) for attempts to model metaphysical explanation as pragmatist explanation.

8 See e.g. Glazier (2017).
} 
2018: 1295). Consequently, in the logical cases there are no grounding-mechanical models at our disposal that explains how the connection runs between the ground and the grounded. Instances of logical grounding are said to be 'bare' - unaccompanied by grounding mechanisms (Trogdon 2018: 1295). ${ }^{9}$

Assuming that grounding mechanisms involve metaphysical determination relations and that logical grounding cannot be accounted for by reference to such relations, the unification gained by modelling explanations that are backed by grounding seems to be contradicted by the disunification of grounding. What are we to do? If we wish to hold on to the mechanical backing claim, there are two options available: (i) deny that there are any instances of bare grounding; or (ii) allow for such instances, but insist that they don't back metaphysical explanation.

With respect to the more radical option (i), a case could be made that there are no instances of bare grounding. It is commonly thought that logical complexity is a feature of representations rather than the world and thus that logical cases target logical priority instead of grounding (Wilsch 2016: 20). On this view, the above claim is false simply because there are no logically complex facts and hence, there can be no grounding-backed explanation of [ $a$ is green and $b$ is blue] in terms of [ $a$ is green] and [ $b$ is blue]. Consequently, putative instances of bare grounding don't pose a threat to grounding-mechanical explanations as there are no such instances to begin with. ${ }^{10}$

The more conservative alternative (ii), and the one that Trogdon opts for, is to allow for instances of bare grounding but to insist that they don't figure in grounding-backed explanations, although they potentially involve other sorts of explanation (2018: 1305). On this view, the above claim is true. However, the instance doesn't back the corresponding explanation for why [ $a$ is green and $b$ is blue] obtains. There are at least two reasons for going in this direction. ${ }^{11}$ One reason is that it might be thought that the kind of explanation that instances of bare grounding back should be conceived of as logical rather than metaphysical. On this view, in explaining why a logically complex fact like [ $a$ is green and $b$ is blue] obtains, one need not refer to worldly facts, but to the propositions or sentences that describe those facts. Perhaps the obtaining of the conjunctive fact can be explained by accounting for how the meaning of the complex sentence ' $a$ is green and $b$ is blue' is built out of the meaning of the atomic sentences ' $a$ is green' and ' $b$ is blue'; or, perhaps we can explain why it is that the conjunctive fact obtains by showing that it is a consequence of the

\footnotetext{
${ }^{9}$ One might also worry that instances of conceptual grounding like:

[ $a$ is a female fox] grounds [ $a$ is a vixen]

are cases of bare grounding. If there are any relations involved here at all, it seems that they would have to be semantic in nature, linking the terms used in the descriptions of the grounding mechanism, rather than the constituents that make up the corresponding facts. See (Chalmers 2012: 464-65; and Smithson 2020: 1504) for discussions about how conceptual explanations can be thought of as being backed by inferential connections between semantically prior and posterior terms.

10 To give up on logical grounding would also, as Trogdon points out (2018: 1301), have the fortunate consequence that various puzzles and paradoxes (Fine 2010; Clark 2015; and Litland 2015) concerning grounding are avoided. See also McSweeney (2020: 166-67).

11 Both of these were suggested to me by Trogdon.
} 
semantics of first-order logic that the truth of a conjunction is a function of the truth of its conjuncts (McSweeney 2020: 159-62). However, neither of these explanations invoke metaphysical determination relations and as such, they fall outside the scope of the grounding mechanism view.

Another reason why one might opt for (ii) is if one thinks that although there is metaphysical explanation in cases of bare grounding, those are cases where grounding doesn't play an essential role in the explanation-bare grounding doesn't back metaphysical explanation. The result is an account of metaphysical explanation that is able to provide us with a unified account only of such explanations which essentially involve grounding - the thought being that in the logical cases, the grounding is incidental to the explanation.

It would thus seem that although grounding mechanics can't say that all metaphysical explanations are grounding-mechanical explanations, they can hold on to the weaker mechanical backing claim. Unfortunately, grounding instances unaccompanied by grounding mechanisms are not limited to logical ground (Trogdon 2018: 1304). To illustrate, consider the fact that Kim's belief is about water $\left(\mathrm{H}_{2} \mathrm{O}\right)$ rather than twin-water (XYZ); or the fact that Kim is able to refer to Oxford University. It is usually thought that the explanations for why these facts obtain are backed by them being, at least partially, grounded in Kim's past causal history-connections that prima facie cannot be cashed out in terms of rules of logic or semantic determination relations, but would have to be accounted for in terms of metaphysical determination relations between constituents of the facts involved (Wilson 2018: 730). If true, at least some of the determination relations that grounding-mechanical explanations track must be diachronic, relating the constituents of facts over time.

This poses a serious threat to the idea that the grounding mechanism view is a unified theory of explanation, as metaphysical determination relations are non-diachronic (Trogdon 2018: 1304). For if true, this would mean that there are groundingbacked metaphysical explanations that don't proceed by virtue of being representations of grounding-mechanisms, thereby refuting the mechanical backing claim. And while it is easy to see how one could bring unity among grounding-mechanical explanation by either redrawing the boundaries of what counts as a metaphysical explanation or by limiting the number of instances that back such explanations, it is difficult to see how either of these responses could handle diachronic ground. Any non-causal explanation of why [Kim's belief is about water] obtains would have to be metaphysical in that it would have to give an account of how the connection runs between facts in the world, at least if we are assuming externalism about the mental (Burge 1979) and causal theories of reference (Kripke 1980 and Putnam 1972). But if so, the explanation in question would have to proceed by reference to grounding mechanisms that invoke metaphysical determination relations between constituents of facts rather than semantic determination relations between sentences that describe those facts. Unfortunately, as it stands, the mechanistic view cannot provide us with such a mechanism. Similarly, it would appear that any metaphysical explanation of why some mental or referential fact obtains would essentially have to make reference to facts in the past if what we want to explain is why this (rather than that) mental or referential fact obtains. So, for example, in providing a metaphysical explanation of why the fact that Kim's belief is about water obtains, it seems that one would have 
to, by virtue of the nature of the explanandum, point to the grounds for [Kim's belief is about water] rather than, say, [Kim's belief is about twin-water]. Of course, the grounding mechanism view is not intended to cover all metaphysical explanations (e.g. essentialist and reductive explanations). But given that instances of diachronic grounding are genuine cases of grounding that back metaphysical explanation but lack corresponding grounding-mechanisms, the mechanistic view is not only an incomplete account of metaphysical explanation, but also an incomplete account of the kind of metaphysical explanation for which it was intended, namely grounding-backed explanation. This is not to say that we cannot provide an explanation of why [Kim's belief is about water] obtains that proceeds in terms of metaphysical laws (Wilsch 2016) or in some other way (see fn. 6). ${ }^{12}$ It is just to say that there are metaphysical explanations backed by grounding and that the grounding-mechanical model doesn't cover them.

To sum up, grounding instances unaccompanied by grounding mechanisms pose a serious threat to the idea that grounding mechanical explanations unify metaphysical explanations backed by grounding. Putative instances of logical grounding can quite easily be dealt with, as one might deny that there are such instances; or accept that there are, but insist that they do not back metaphysical explanation. Unfortunately, instances of diachronic ground are not as easily dealt with. Not only do they seem to be genuine instances of ground that back metaphysical explanation, they also seem to play an essential role in explaining why certain facts obtain. One of the central tasks for the grounding mechanic is thus to give an account of how metaphysical explanations backed by instances of diachronic ground can be made to fit the mechanistic view. In Sect. 4., I will develop a solution to the problem of diachronic ground. But before I do, we have to discuss the second concern and how it relates to the first.

\section{Grounding Mechanisms Unaccompanied by Relevant Determination Relations}

The second concern is that the grounding mechanism view (as stated) allows for grounding mechanisms that are unaccompanied by relevant determination relations. Trogdon asks us to consider the following case (Trogdon 2018: 1301): suppose that, while $\{$ Socrates $\}$ lacks property $\mathrm{P}$,

(1) [Socrates exists] grounds [Socrates exists or $\{$ Socrates $\}$ has P].

In this case, a constituent of the grounding fact (i.e. Socrates), stands in the metaphysical determination relation of set formation to a constituent of the grounded fact (i.e. $\{$ Socrates\}). It follows that the grounding relation is an instance of a grounding

\footnotetext{
12 Trogdon's own view is that the diachronic cases can potentially be metaphysically explained by means of metaphysical subsumption, i.e. by representing metaphysical laws in conjunction with facts as logically entailing the explanandum fact (2018: 1305). Unfortunately, he does not develop this thought further.
} 
mechanism. The problem is that by representing the grounding mechanism thus we don't exhibit how the connection runs between the ground and the grounded. Any explanation of why the grounded fact obtains would presumably involve something like disjunction introduction rather than set formation. And a rule like disjunction introduction is not a metaphysical determination relation.

Trogdon thinks that the problem can be avoided by adding a further condition on what it is to be a mechanistic model of grounding-one that concerns the form that grounding relations are represented as having. The idea is that in addition to veridically representing a metaphysical determination relation as being essentially connected to the Grounding Condition, the mechanistic model should also represent the grounding claim as having the same form as the grounding relation that the metaphysical determination relation is essentially connected with (Trogdon 2020: 1302). And in the above case, there is no such match. Thus, the form of the grounding claim that set formation is essentially connected with is " $[x$ exists $]$ grounds $[y$ exists $]$ " whereas the form of the above grounding claim is " $[x$ exists $]$ grounds $[x$ exists or $y$ has such-and-such property]". Given the additional constraint, the model isn't mechanistic.

The problem is that the formal condition excludes too much. Consider the following:

\section{(2) [Socrates exists] grounds [ $\{$ Socrates $\}$ exists or $\{$ Socrates $\}$ exists $]$.}

Here the grounding claim is of the form " $[x$ exists $]$ grounds [ $y$ exists or $y$ exists]" whereas the grounding claim that set formation is essentially connected with is of the form " $[x$ exists $]$ grounds [ $y$ exists $]$ ". In effect, $(2)$ shouldn't count as a mechanistic model. Yet, there is nothing more to the explanation of why the disjunctive fact obtains than there being a relation of set formation holding between the philosopher and the singleton. Intuitively, this should suffice to make (2) mechanistic. After all, the grounding stated is an instance of a grounding mechanism and the model correctly represents this by exhibiting how the connection runs between the fact involving Socrates and the corresponding disjunctive fact involving the singleton. What is more, since metaphysical determination relations are irreflexive (Trogdon 2018: 1292) and [\{Socrates $\}$ exists] is identical to [\{Socrates $\}$ exists or \{Socrates $\}$ exists], there is no chain of metaphysical determination relations linking the philosopher to the singleton and some other determination relation, in turn, relating the singleton to some constituent of the disjunctive fact. We are thus not in a position to argue that the grounding claims at the different connecting stages will be of the same form as the grounding claims that the corresponding determination relations are essentially connected with at those stages. ${ }^{13}$

\footnotetext{
13 It is easy to see how one could generate similar counterexamples by simply replacing the description of the grounded fact in the model with a formally different description of the same fact. Suppose that: [Socrates exists] grounds [ $\{$ Socrates $\}$ exists and 〈\{Socrates $\}$ exists〉 is true], and that some sort of identity theory is true, according to which [ $\langle\{$ Socrates $\}$ exists $\rangle$ is true] and [ $\{$ Socrates $\}$ exists] is the same fact. If so, then there is nothing more to the explanation of why the disjunctive fact obtains than there being a relation of set formation between Socrates and \{Socrates
} 
The most straightforward response is to deny that disjunctive facts are identical to their disjunct fact and so, that [\{Socrates $\}$ exists or \{Socrates $\}$ exists] and [\{Socrates $\}$ exists] are two distinct facts. In that case, the explanation will be merely partially mechanistic; the idea being that we appeal to set formation as the mechanism that mediates the link between the fact that Socrates exists and the fact that his singleton exists, and then appeal to whatever the connection is in the logical cases to get us from the fact that singleton Socrates exists to the corresponding disjunctive fact.

But this is not a plausible solution to the problem at hand. In addition to turning the grounding mechanistic explanation into merely partially mechanistic explanation, the solution goes against the conception of facts that grounding-mechanical explanations demand. Roughly speaking, there is the conception of facts as 'worldly' according to which facts have worldly constituents (i.e. objects and properties) and there is the conception of facts as 'conceptual' according to which the constituents of facts are representations or modes thereof. On a conception of facts as conceptual, which fact is referred to by means of descriptions like "[P]" is highly sensitive to the concepts involved in the descriptions, whereas on a worldly conception of facts, there is significantly less sensitivity in how the facts are described, as they are taken to be individuated solely by their worldly constituents and the manner of their combination (cf. Correia 2010: 256-59). Now, since the determination relations that characterize grounding mechanisms relate properties and objects (as opposed to representations), the grounding mechanism view is committed to a worldly conception of the nature of facts. ${ }^{14}$ Thus, the grounding mechanic is obligated to view $[\{$ Socrates $\}$ exists or $\{$ Socrates $\}$ exists $]$ and [\{Socrates $\}$ exists $]$ as one and the same fact.

But if so, there is a single determination relation at work here, namely set formation, holding between the philosopher and his singleton. What is the grounding mechanic to do? Well, notice that although the facts themselves are worldly entities, the models are representations. This suggests that there might be a way for the grounding mechanic to avoid the above problem by adding something to the characterization of mechanistic models. Something along the following lines: for a representation of a fact to be included in a mechanistic model, the fact is to be described with its "canonical representation". ${ }^{15}$ Going with this option, one could argue that the disjunctive representation isn't a canonical representation and so, that the corresponding model isn't mechanistic.

To make it into a mechanistic model one would have to describe the fact denoted by "[\{Socrates $\}$ exists or $\{$ Socrates $\}$ exists $]$ " in its canonical form, in which case we get:

(3) [Socrates exists] grounds [ $\{$ Socrates $\}$ exists $]$,

\footnotetext{
14 Trogdon explicitly states that he has a worldly conception of facts and that he assumes that they are "a distinctive kind of entity" (2018: 1289-90n).

15 Trogdon suggested this modification to me in a private conversation.
} 
assuming that "[\{Socrates $\}$ exists $]$ " is the canonical representation of the fact denoted by the disjunctive description. One can then easily provide a mechanistic explanation of why the grounded fact obtains by reference to the relation of set formation that holds between Socrates and \{Socrates\}.

But again, this isn't an attractive strategy, as in addition to increasing the complexity of the view, the modification requires us to specify just what it is to be a canonical representation of a fact. This is not an easy task. The representations would have to be unique; they would have to avoid arbitrariness in the choice of descriptions; and they would have to be decisive for all veridical descriptions of facts. I fail to see how these desiderata could ever be fulfilled in a specification of what it is for a representation of a fact in a mechanistic model to be in its canonical form. Granted, it is reasonable to think that the choice of description will not be arbitrary in the sense that the canonical representations will most likely fall out of our initial choices concerning how to regiment grounding discourse on the basis of various pragmatic considerations (e.g. avoiding needless complexity) that guide regimentation. But, if true, this merely goes to show that pragmatic factors have a role to play in deciding how to represent grounding discourse. It does not show that pragmatic factors are decisive in whether a model is a representation of a fact that can be given a grounding-mechanical explanation, let alone that a further restriction on what it is for a fact to be in its "canonical form" has a bearing on whether the model is mechanistic or not. The modification deems (3) mechanistic, but not (2). But why should the way (2) describes the grounded fact be any less "mechanistic" (whatever that means) than the way (3) describes it? After all, they are both descriptions of the same fact and they seem worthy of the epithet "mechanistic" simply because they both model an instance of a grounding mechanism. To respond to this by saying that (2) is not mechanistic because the grounded fact is not represented in its canonical form is an ad hoc response that goes against the idea that mechanistic models should allow for different syntactical descriptions of the same fact. ${ }^{16}$ The modification is only there to avoid instances like (2) to refute the formal condition. It serves no other purpose.

Perhaps there are other ways to modify the formal condition that are less ad hoc and that allow for different ways of representing the same underlying facts. If so, I'm not aware of them. Be that as it may, one might plausibly question the need for a formal condition on the characterization of mechanistic models to begin with. For notice that there is only a need for such a condition if (a) there are genuine instances of logical grounding; and (b) instances of logical grounding back metaphysical explanation. Needless to say, if either one of these is false, then there is no need for a grounding-mechanical model that tells us how the connection runs between more fundamental atomic facts and the less fundamental, but logically

\footnotetext{
16 As a matter of fact, it is commonly thought that not only should a single model allow for syntactically different descriptions of the same fact but also that mechanical descriptions are semantic entities in that there can exist many syntactically different formulations of the same description (Glennan 2005). On this view, not only do (4) and (5) depict the same model, the corresponding descriptions are in fact identical by virtue of being semantically equivalent.
} 
more complex, facts that the former allegedly ground. If (a) is false and there are no instances of logical ground, then statements like (1), that pose a threat to the grounding mechanic, are false. [Socrates exists] doesn't ground [Socrates exists or \{Socrates\} has P] because there are no logically complex facts. If, on the other hand, (b) is false, then (1) might still be true-it is just that it doesn't back the explanation for why the logically complex fact obtains: either because the explanation isn't metaphysical; or it is, but the grounding instance doesn't play an essential role in the explanation. Irrespective of whether (a) or (b) is false, logical grounding falls outside the scope of what is covered by grounding-mechanical explanations. And either way, there is no need for a grounding-mechanical model, let alone for a formal condition on the characterization of such models. If (a) is true and (b) is false, the grounding relation depicted by (1) would still be an instance of a grounding mechanism. However, by providing a model of it, we don't explain why [Socrates exists or \{Socrates\} has P] obtains by reference to [Socrates exists] when \{Socrates \} lacks P. But this isn't a problem. The mechanical backing claim only excludes explanations that are backed by grounding but that don't proceed by being representations of grounding mechanisms. This is fully compatible with there being instances of grounding mechanisms like (1) that fail to explain the grounded fact. It is just that any metaphysical explanation that is backed by grounding proceeds by reference to such mechanism.

This is not the place to go into a detailed discussion concerning which of (a) or (b) is false. Suffices it for present purposes to recall that either of them must be false if the grounding mechanic is to hold on to the mechanical backing claim. The point of the present section has been to show that there are independent reasons to reject at least one of them. For to accept both would not only lower the grounding mechanics' chances of ever having a unified theory of metaphysical explanation, it would also require a formal condition on what it is to be a mechanistic model-a condition that, when implemented, raises more questions than it answers.

\section{Diachronic Grounding and the Contingency of Grounding}

Setting the problem of logical grounding to the side, let us turn to the problem of diachronic grounding and the threat it poses to the idea that we are able to unify grounding-backed metaphysical explanations by taking such explanations to be representations of grounding mechanisms. The threat is much more severe than the one posed by alleged cases of logical grounding in that the diachronic cases seem to be not only genuinely metaphysical but also essential in explaining why the members of a fairly large and important set of facts obtain (e.g. various mental and referential facts). Recall that the problem is how to represent diachronic grounding as instances of grounding mechanisms, given that such mechanisms solely involve metaphysical determination relations that are non-diachronic. Of course, one could simply drop this requirement, allowing for grounding mechanisms involving metaphysical determination relations that relate over time. I take it, however, that most grounding theorists would not be willing to take this route as it is hard to see just what these 
relations could possibly be. It seems that the diachronic relations of relevance (e.g. causation) doesn't satisfy the Grounding Condition (Trogdon 2018: 1304).

In view of this, one might adopt the Lucretian strategy of grounding past-dependent facts in presently existing facts consisting of past-tensed properties. This would enable us to say that [Kim's belief is about water] is grounded in the fact that Kim is presently such as to have causally interacted with $\mathrm{H}_{2} \mathrm{O}$ - thereby not having to give up the idea that metaphysical determination relations do not extend over time while simultaneously opening up for the possibility of having a grounding-mechanical explanation of why [Kim's belief is about water] obtains. There are, however, reasons to suggest that there are no such past-directed and extrinsic properties (Sider 2001; and Merricks 2007). Intuitively, if grounding is non-diachronic, then any property explaining why [Kim's belief is about water] obtains at time $t$ would have to make a difference to the intrinsic nature of Kim at that time. A property like being such as to have causally interacted with $\mathrm{H}_{2} \mathrm{O}$ doesn't, and so, isn't part of what grounds [Kim's belief is about water] at $t$ (Cameron 2010). ${ }^{17}$ Another solution is to make a distinction between 'broad' and 'narrow' mental content and then argue that only mental facts involving the latter are fit to be grounded. On this view, what is grounded is not the fact that Kim's belief is about water, but the corresponding narrow content surrogate of that fact. Since the content of such a fact is not a function of past facts, it might be thought that we are able to explain the obtaining of [Kim's belief is about water] solely in terms of non-diachronic determination relations. However, the very notion of narrow content remains controversial (Adams et. al. 1990; and Saidel 1994) and there is reason to think that it is only the broad contents of our beliefs that are relevant when explaining why some functionally realized mental fact obtains (cf. Williamson 1995).

So, what are we to do? Here's a suggestion: recognize that some mechanical explanations are backed by grounding instances that are contingent on a specific set of circumstances such that $[\mathrm{P}]$ might be grounded in some plurality of facts $\Delta$ in circumstances $\mathrm{S}$ and, yet, $[\mathrm{P}]$ does not obtain in the presence of $\Delta$ under a different set of circumstances $S^{*}$ (Skiles 2015). To illustrate, let $C$ be the property of having some specific crystalline structure and let $a$ be some piece of brittle glass. Now, suppose that:

(4) [ $a$ has C] grounds [had $a$ been dropped it would have shattered].

is true in the actual circumstances where the glass is held $5 \mathrm{~m}$ above a concrete floor and subject to a strong enough gravitational force to shatter upon impact. In some other set of circumstances, where $a$ is held a couple of centimeters above a soft mattress or in space where $a$ is subject to no net gravitational force, (4) comes out false. Here's another example: suppose that $m_{1}, m_{2} \ldots m_{\mathrm{n}}$ are all the men there are and that:

(5) $\left[m_{1}\right.$ is a mortal man], [ $m_{2}$ is a mortal man], ...[ $m_{\mathrm{n}}$ is a mortal man] grounds [all men are mortal],

\footnotetext{
${ }^{17}$ One could also take past-dependent facts to be grounded in facts involving temporal distributional properties (TDP) (see Parsons 2004; and Cameron 2010). This would avoid the above problem as TDP make an intrinsic difference to the objects that instantiate them. However, this solution has its own problems (Corkum 2014) and has the unfortunate consequence that grounding theorists that want to provide mechanistic explanations of the grounding of mental and referential facts are committed to a specific and controversial view of properties.
} 
is true. As Bertrand Russell (1956) thought us, the large plurality of facts consisting of mortal men will only ground the fact that all men are mortal in circumstances like the actual where there are no men in addition to $m_{1}, m_{2} \ldots m_{\mathrm{n}}$. Under a different set of circumstances where all of $m_{1}, m_{2} \ldots m_{\mathrm{n}}$ exist, the presence of some immortal man will make (5) false.

By recognizing that instances of diachronic ground are contingent in the above sense, we can give a mechanistic explanation for why various mental and referential facts obtain, without having to resort to diachronic determination relations, suspicious properties or narrow content. Again, consider [Kim's belief is about water]. Since it is not a fundamental fact, it requires to be grounded in something more fundamental. However, there is no reason in particular why we should include into the more fundamental plurality of facts the various spatiotemporally scattered facts that make Kim's belief about water rather than twin-water. This is not to say that we can only provide grounds for mental facts involving narrow content or that mental facts whose contents are broad are not fit to be grounded. For we can now say that mental facts involving broad contents are never grounded simpliciter, but always under a specific set of circumstances, namely those that must hold for the mental fact to obtain. In view of this, what grounds the fact that Kim's belief is about water is not some spatiotemporally scattered plurality of facts involving past facts about Kim's interactions with $\mathrm{H}_{2} \mathrm{O}$. To see why, suppose that mental facts are grounded in neurophysiological facts (perhaps, together with other non-mental facts). Suppose furthermore that mental properties are functionally realized by neurophysiological properties and that the Grounding Condition is an essential truth of functional realization. It is thus part of what it is to be functional realization that if being in brain state $n$ functionally realizes having belief $b$ when $x$ has both properties, then $[x$ is in brain state $n]$ is among some plurality of facts that grounds $[x$ has belief $b]$. So, let [Kim is in brain state $\varphi$ ] be the neurophysiological fact that grounds [Kim's belief is about water]. Since [Kim is in brain state $\varphi$ ] will not be among some plurality of non-mental facts $\Gamma$ such that $\Gamma$ grounds [Kim's belief is about water] unless in circumstances like the actual, it follows that the grounding mechanism that explains why [Kim's belief is about water] obtains involves having a belief about water being functionally realized by being in brain state $\varphi$. The neurophysiological property will only functionally realize the mental property in circumstances like those found here on Earth where the subject of the belief has causally interacted with $\mathrm{H}_{2} \mathrm{O}$. In circumstances like those found on Twin-Earth where the subject has causally interacted with XYZ, the same neurophysiological property will functionally realize having a belief about twin-water. In either case, the grounding mechanism does not involve constituents of facts that stand in diachronic determination relations to constituents of the corresponding mental fact, as the former constituents are not part of the mechanism itself but part of the circumstances that enables the grounded fact to obtain. ${ }^{18}$

\footnotetext{
18 Similarly, the past causal facts that enables Kim to refer to Oxford University, rather than something else with the same name, need not be thought of as part of the mechanism that explains why [Kim is able to refer to Oxford University] obtains but part of the circumstances that are necessary for the explanandum fact to obtain.
} 
Since some grounding instances are contingent on circumstances external to the mechanisms and grounding mechanical models work by representing instances of grounding mechanisms, some mechanical explanations of the obtaining of facts will depend on factors that are not represented by the model itself. This might be taken to suggest that mechanical explanations of such facts are in some sense incomplete. We don't have a complete grounding-backed explanation of why a fact obtains unless the underlying grounding facts metaphysically necessitate the grounded fact. Thus, the thought goes, the grounding mechanism that explains why [Kim's belief is about water] obtains must be supplemented with facts about Kim's causal history. Only then would we be in a position to argue that we have a complete explanation of [Kim's belief is about water].

Given that we have a complete explanation of why $[\mathrm{P}]$ obtains in virtue of $\Delta$ only if $\Delta$ fully grounds [P], the objection rests on the assumption that $\Delta$ is not [P]'s full ground unless the former metaphysically necessitates the latter. But there are no non-question begging reasons to think that this is true. For when $\Delta$ alone fails to metaphysically necessitate $[\mathrm{P}]$, but it is impossible in circumstances $\mathrm{S}$ for $\Delta$ to obtain in the absence of $[\mathrm{P}]$, we can say that $\Delta$ fully grounds $[\mathrm{P}]$ in $\mathrm{S}$. Depending on the metaphysical determination relations involved in the grounding of mental facts, the neurophysiological fact will either be sufficient on its own to fully ground the mental fact in the relevant circumstances; or the neurophysiological fact is among some plurality of facts that fully grounds the mental fact under those circumstances. So, for example, if the grounding of the mental in the physical is underpinned by determinate-determinable relations (Yablo 1992; and Shoemaker 2001) then [Kim's being in brain state $\varphi$ ] is, in circumstances like those found on Earth, by itself sufficient to fully ground the fact that Kim's belief is about water. If, on the other hand, the physical-mental bond is better spelt out in terms of functional realization or some other determination relation that underlie partial grounding, then [Kim's being in brain state $\varphi$ ] will not suffice on its own but would have to be among some plurality of facts $\Gamma$ that, in the circumstances in question, fully grounds the fact that Kim's belief is about water. ${ }^{19}$

\footnotetext{
19 Notice that we cannot make do without metaphysical determination relations that link constituents of grounding fact(s) that merely partially ground the fact they ground by replacing a partial grounding characterization like:

if $x$ stands in relation $\mathrm{R}$ to $y$, then there is a fact $\mathrm{A}$ and a fact $\mathrm{B}$ where $\mathrm{A}$ is among a plurality of facts $\Gamma$ such that $\Gamma$ grounds $B$.

with corresponding contingentist characterizations like:

if $x$ stands in relation $\mathrm{R}$ to $y$ then there is a fact A and a fact B such that A (fully) grounds B in circumstances $\mathrm{S}$,

by letting the facts that make up the plurality (with the exception of A) constitute the circumstances $\mathrm{S}$. The reason for this is straightforward. Some metaphysical determination relations (e.g. constitution, functional realization and mereological realization) are, by virtue of their nature, such that they only relate constituents of grounding facts that are among some plurality of facts (see Sect. 1. above). The facts that comprise the plurality cannot be thought of as part of the circumstances as it is essential to the metaphysical determination relations in question that they relate constituents of facts that, only when taken together with other facts, fully grounds the grounded fact.
} 
If this is correct, then that $\varphi$ exists and $[\mathrm{P}]$ does not is fully compatible with $\Delta$ being [P]'s full ground. The only reason to think that the relevant circumstances in which $\Delta$ fully grounds $[\mathrm{P}]$ must be added to the plurality of grounding facts, is if one takes it to be a necessary condition that full grounds necessitate what they ground. But this is exactly what the grounding contingentist denies. Thus, to demand that the facts that make up the circumstances must be included in the grounding facts is to beg the question (cf. McBride 2021). Furthermore, it does not in general follow that if $\Delta$ grounds $[\mathrm{P}]$ only in circumstances $\mathrm{S}$, then $\Delta$ must be supplemented by the facts that make up $\mathrm{S}$ for $\Delta$ to fully ground [P]. So, for example, [Socrates exists] does not ground [\{Socrates $\}$ exists] unless the circumstances are such that the cosmological constant $\Lambda$ is roughly $2.036 \times 10^{-35} \mathrm{~s}^{-2}$. Had the value been slightly different, then nothing would have existed to ground the fact that singleton Socrates exists. But this does not mean that [Socrates exists] has to be supplemented with anything, let alone with $\left[\Lambda \approx 2.036 \times 10^{-35} \mathrm{~s}^{-2}\right.$, in order for [Socrates exists] to fully ground [\{Socrates $\}$ exists]: the fact that Socrates exists suffices by itself. Finally, the facts that make up the circumstances in which some plurality of facts $\Delta$ grounds [P], need not be more fundamental than $[\mathrm{P}]$. There is, for example, no reason to think that the facts that describe the relevant circumstances where (4) is true are more fundamental than the fact that had $a$ been dropped it would have shattered. However, if $\Delta$ grounds [P], then each fact in $\Delta$ needs to be more fundamental than [P]. Not only is there no requirement that the circumstances be added to the plurality of grounding facts, there are grounding instances where the facts that make up those circumstance cannot, even in principle, be added. Unless further argument is provided, we can thus hold on to the view that $\Delta$ fully grounds [P] even when it is possible for $\Delta$ to exist when $[\mathrm{P}]$ does not. Until then, there are no non-question begging reasons to think that explanations backed by contingent grounding instances are not complete.

Before closing this section, let me just briefly discuss another way in which one might think that metaphysical explanations backed by contingent grounding are incomplete. Such explanations, one might argue, allow for grounding mechanisms that in circumstances $S$ explain the obtaining of $[P]$ and, yet, in circumstances $S^{*}$, do not explain [P] but rather [Q]. For if true, this would mean that the mechanistic model above would not give an explanation for why the fact that Kim's belief is about water obtains, as the same grounding mechanism could, in a different set of circumstances, explain the obtaining of the fact that Kim's belief is about twinwater. Fortunately, it isn't true. To see why, notice that when $\Delta$ does not ground $[\mathrm{P}]$ unless in circumstances $\mathrm{S}$, then $\mathrm{S}$ is necessary for $\Delta$ to ground $[\mathrm{P}]$ in that had $\mathrm{S}$ not obtained, $[\mathrm{P}]$ would not have obtained for $\Delta$ to ground. Circumstances like the actual are thus necessary for [Kim's being in brain state $\Delta$ ] to ground [Kim's belief is about water]. But if so, then in the relevant circumstances, the mental property that is metaphysically determined by being in brain state $\varphi$ cannot be having a belief about twin-water, but must be having a belief about water. Consequently, since the instances of metaphysical determination relations that make up grounding mechanisms have their identity, at least in part, determined by the terms they relate, a grounding mechanism that accounts for the connection between [Kim is in brain state $\varphi$ ] and [Kim's belief is about twin-water] will be different from a mechanism that depicts the connection between [Kim is in brain state $\varphi$ ] and [Kim's belief is 
about water], even when the metaphysical determination relations involved are of the same type. The grounding mechanism will thus not be the same on Earth as on Twin-Earth, as the relevant circumstances at hand are necessary for the corresponding grounding instances to occur in the first place. ${ }^{20}$

\section{Summary}

In this paper I have addressed two concerns with the grounding mechanism view as put forth by Trogdon. The first concern is that there are examples of grounding instances that are unaccompanied by grounding mechanism, which refutes the grounding mechanical claim and diminishes the prospects of the grounding mechanic ever having a unified theory of metaphysical explanation. The second concern is that Trogdon's particular take on instances of bare grounding paves the way for grounding mechanisms that are unaccompanied by (relevant) determination relations. Trogdon thinks that such cases don't undermine the view once a formal condition is placed on mechanistic models. I have argued that the formal condition excludes too much and that it is hard to see how this could be avoided within a mechanistic framework. I ended the paper by arguing that while we have reason to think that logical grounding isn't covered by grounding-mechanical explanations, we can easily accommodate diachronically grounded facts. The solution is to take the grounding of such facts to be contingent on the circumstances in which the grounding takes place and to view those circumstances as preconditions for the explanandum fact to obtain.

Acknowledgements I would like to thank an anonymous referee for their comments. I also wish to thank Kelly Trogdon for his insightful and invaluable comments on an earlier draft of this paper. I am also indebted to the participants at the higher seminar in theoretical philosophy at Lund University.

Funding Open access funding provided by Lund University.

Open Access This article is licensed under a Creative Commons Attribution 4.0 International License, which permits use, sharing, adaptation, distribution and reproduction in any medium or format, as long as you give appropriate credit to the original author(s) and the source, provide a link to the Creative Commons licence, and indicate if changes were made. The images or other third party material in this article are included in the article's Creative Commons licence, unless indicated otherwise in a credit line to the material. If material is not included in the article's Creative Commons licence and your intended use is not permitted by statutory regulation or exceeds the permitted use, you will need to obtain permission directly from the copyright holder. To view a copy of this licence, visit http://creativecommons.org/licen ses/by/4.0/.

\footnotetext{
20 To deny this one would have to think that neurophysiological properties realize narrow mental content properties that somehow remain invariant when going from one set of circumstances to the next. But, as already noted, it remains contentious to claim that there are such properties.
} 


\section{References}

Adams F, Drebushenko D, Fuller G, Stecker R (1990) Narrow content: fodor's folly. Mind Lang 5(3):213-229

Baron S, Norton J (2021) Metaphysical explanation: the kitcher picture. Erkenntnis 86:187-207

Burge T (1979) Individualism and the mental. In: French PA, Uehling TE, Wettstein HK (eds) Midwest studies in philosophy, 4, Minneapolis: University of Minnesota Press

Cameron R (2010) Truthmaking for presentists. Oxford Studies in Metaphysics 6:55-100

Chalmers D (2012) Constructing the World. Oxford University Press, Oxford

Clark M (2015) A puzzle about partial grounding. Thought 4:189-197

Corkum P (2014) Presentism, truthmakers and distributional properties. Synthese 191(14):3427-3446

Correia F (2010) Grounding and truth-functions. Logique Et Anal (NS) 53(211):251-279

Craver CF (2007) Explaining the Brain: Mechanisms and the Mosaic Unity of Neuroscience. Clarendon Press, Oxford

Fine K (2010) Some puzzles of ground. Notre Dame. J Formal Logic 51:97-118

Glazier M (2017) Essentialist explanation. Philos Stud 174(11):2871-2889

Glennan SS (2005) Modeling mechanisms. Studies in the History and Philosophy of the Biological and Biomedical Sciences 36:375-388

Koslicki K (2015) The coarse-grainedness of grounding. In: Bennett K, Zimmerman D (eds) Oxford studies in metaphysics, vol 9. Oxford University Press, Oxford

Kovacs D (2020) Metaphysically explanatory unification. Philos Stud 177:1659-1683

Krämer S (2013) A simpler puzzle of ground. Thought 2(2):85-89

Kripke S (1980) Naming and Necessity. Harvard University Press, Cambridge MA

Levine J (2001) Purple Haze. Oxford University Press, Oxford

Litland JE (2013) On some counterexamples to the transitivity of grounding. Essays Philos 14(1):19-32

Litland JE (2015) Grounding, explanation and the limit of internality. Philos Rev 124:481-532

Machamer PK, Darden L, Craver CF (2000) Thinking about mechanisms. Philos Sci 67:1-25

Maurin A-S (2019) Grounding and metaphysical explanation: it's complicated. Philos Stud 176(6):1573-1594

McBride F (2021) Truthmakers. In: Zalta EN (ed) Stanford encyclopedia of philosophy. https://plato.stanf ord.edu/entries/truthmakers/

McSweeney M (2020) Debunking logical ground: distinguishing metaphysics from semantics. J Am Philos Assoc 6(2):156-170

Merricks T (2007) Truth and Ontology. Clarendon Press, Oxford

Miller K, Norton J (2017) Grounding: it's all in the head. Philos Stud 174(12):3059-3081

Parsons J (2004) Distributional properties. In: Jackson F, Priest G (eds) Lewisian themes. Oxford University Press, Oxford, pp 173-180

Putnam H (1972) The meaning of 'meaning.' Minn Stud Philos Sci 7:131-193

Raven M (2013) Is ground a strict partial order? Am Philos Q 50(2):191-199

Rodriguez-Pereyra G (2015) Grounding is not a strict order. J Am Philos Assoc 3:517-534

Russell B (1956) "Logical Atomism" in Logic and Knowledge. Allen and Unwin, London, pp 323-343

Saidel E (1994) Discussion: content and causal powers. Philos Sci 61:658-665

Schaffer J (2010) Monism: the priority of the whole. Philos Rev 119:31-76

Schaffer J (2012) Grounding, transitivity and contrastivity. In Correia F, Schnieder B (eds) Metaphysical grounding: understanding the structure of reality. Cambridge University Press, Cambridge, pp 122-138

Shapiro S (1997) Philosophy of Mathematics: Structure and Ontology. Oxford University Press, Oxford

Shoemaker S (2001) Realization and mental causation. In: Proceedings of the 20th world congress in philosophy. Philosophy Documentation Center, Cambridge, pp 23-33

Sider T (2001) Four-Dimensionalism: An Ontology of Persistence and Time. Oxford University Press, Oxford

Skiles A (2015) Against grounding necessitarianism. Erkenntnis 80(4):717-751

Smithson R (2020) Metaphysical and conceptual grounding. Erkenntnis 85(6):1501-1525

Tahko T (2013) Truth-grounding and transitivity. Thought 2(4):332-340

Thompson N (2019) Questions and answers: metaphysical explanation and the structure of reality. J Am Philos Assoc 5(1):98-116

Trogdon K (2018) Grounding-mechanical explanation. Philos Stud 175(6):1289-1309

Trogdon K (2020) Truthmaking. In: Raven MJ (ed) Routledge Handbook of Metaphysical Grounding. Routledge, New York, pp 396-407

Williamson T (1995) Is knowing a state of mind? Mind 104(415):533-565 
Wilsch T (2016) The deductive-nomological account of metaphysical explanation. Australas J Philos 94(1):1-23

Wilson A (2018) Metaphysical causation. Noûs 52(4):723-751

Wilson J (2016) The unity and priority arguments for grounding. In: Aizawa K, Gillett C (eds) Scientific composition and metaphysical ground. Palgrave, London

Yablo S (1992) Mental causation. Philos Rev 101(2):245-280

Publisher's Note Springer Nature remains neutral with regard to jurisdictional claims in published maps and institutional affiliations. 